\title{
Comprehensive Research on Application and Optimization of Heat Storage Technology Under Different Industrial Demand: Based on Medium and Low Temperature
}

\author{
Zhenrui Zhang*
}

Zhejiang University Ningbo Institute of Technology (NIT), Ningbo 315000, Zhejiang Province, China. E-mail: 2231315705@qq.com

Abstract: Heat storage technology is one of the key technologies in the field of solar thermal power generation and cogeneration. It uses heat storage materials as the media to store solar thermal energy, industrial waste heat, low-grade waste heat and other kinds of thermal energy, and release it when needed, so as to solve the mismatch between energy supply and demand. This paper introduces the classification and characteristics of heat storage technology, analyzes the research progress of heat storage technology in the fields of solar thermal power generation, heat storage materials and industrial drying, and forecasts the development trend of heat storage technology in the future. The results indicate that heat storage technology is beneficial to improve industrial production efficiency, broaden industrial energy conservation and emission reduction ideas, and has a great application prospect under the continuous improvement of practitioners with innovative spirit.

Keywords: Heat Storage Material; Solar Thermal Power Generation Technology; Phase Change Material; Heat Storage Tank; Single Tank Heat Storage; Double Tank Heat Storage; Industrial Drying; Inter-Seasonal Heat Storage

\section{Adjustment and optimization of solar thermal storage technology under the demand of solar thermal power generation}

\subsection{Requirements introduction}

Solar thermal power generation technology was faced with the problem that the output of electric energy cannot be produced continuously and steadily from the beginning of the application. And this is because of the intermittent and unsteady solar irradiance. The situation was initially improved with a two-tank heat storage scheme which is mainly composed of a cold tank and a heat tank through a simple endothermic and exothermic cycle. Although most solar thermal power generation and heat storage systems have adopted this solution up to now, both tanks are required to have the capacity to provide storage space for the entire medium, which results in producing some empty unused volume in the tank during operation, and the high cost caused by structural waste is one of the obstacles to the replacement of thermal power model ${ }^{[1]}$.

\subsection{Solutions}

To solve this problem, an improved scheme combining single-tank heat storage with solar energy-assisted coal-fired power stations is proposed. Compared with the double-tank technology, it does not need to add circula-

Copyright (C) 2020 Zhenrui Zhang

doi: 10.18282/pef.v9i2.1093

This is an open-access article distributed under the terms of the Creative Commons Attribution Non-Commercial License

(http://creativecommons.org/licenses/by-nc/4.0/), which permits unrestricted non-commercial use, distribution, and reproduction in any medium, provided the original work is properly cited. 
tion pump and nitrogen protection device in the heat storage medium, which greatly simplifies the structure and reduces the cost. Especially for the double-tank heat storage system of oil-salt heat exchanger, the advantages of a single tank system of the same size are apparent.

\subsection{Single-tank thermocline theory}

The single-tank heat storage technology is based on the theory of the inclined temperature layer. It can get the heat moved from the end of endothermic to the end of exothermic by controlling the movement of the inclined temperature layer. In order to improve the effect of the inclined temperature layer, the tank should be filled with porous media, and the single-tank inclined-temperature-layer system can be divided into two types: the direct contact system and the indirect contact system.

Several researches have been done on single-tank heat storage system at home and abroad. Some of the conclusions are as follows: refractory ceramics should be selected as solid materials for heat storage; The experimental results of Laing et al. show that metal debris can be added into the heat transfer medium for enhanced heat transfer. The CFD numerical simulation method of Han Jingxiao et al. shows that the plate heat storage structure has obvious comprehensive advantages, the mass flow rate in the heat storage structure, the Height and cross-sectional area of the heat storage unit have great influence on the heat storage performance ${ }^{[2]}$.

Recent innovations in the field of medium and low temperature heat storage include, but are not limited to, the above contents. It can be seen that with the maturity of the technology, researchers in the field of large-scale solar power generation are more focused on the innovative research of single-tank heat.

\section{Research achievements of low temperature heat storage materials}

The functional thermal fluid is a new material for heat storage and transfer, and its application research is in the ascendant in developed countries. Latent functional heat fluid is a kind of multiphase fluid which is composed of single phase heat transfer fluid and phase change microcapsule. Compared with the ordinary single-phase heat transfer fluid, the apparent specific heat capacity of the heat transfer medium can be greatly in- creased in the phase transition zone, which can theoretically enhance the heat transfer.

Some functional thermal fluids mixed by ordinary materials have become the research focus in the field of thermal energy storage in some developed countries such as the United States, Japan and Germany. These materials usually have the following characteristics: high heat storage density, high heat transfer rate, wide application temperature range, heat storage, heat transfer process heat loss is small. They can reduce the size of the heat exchanger and the corresponding pipeline and can be divided into five types: ice-water mixture, latent heat microemulsion, latent heat seal packaging material, cage-type inclusion compound, and shaped phase change heat storage material ${ }^{[3]}$.

The potential applications of functional thermal fluids are wide ranging, but they mainly focus on the areas of building heating, hot water supply, air conditioning systems and other energy storage. With the deepening of the research, its application is developing to a wide range and depth, which is worthy of attention researchers in the field of energy storage in China.

\subsection{Cross-season heat storage technology}

And there is a long-term heat storage area for the collection of heat in spring, summer and autumn for winter heating. Compared with short-term heat storage, it needs to improve the system's ability of long-term and stable heat storage. Solar Energy inter-seasonal heat storage technology mainly includes sensible heat storage, latent heat storage and thermal chemical heat storage, among which sensible heat storage technology has been widely used, it can be divided into four types: water tank heat storage, rock heat storage, buried pipe heat storage, and underground aquifer heat storage. Zhao Xuan et al. summarized the achievements in the field of sensible heat storage in recent years ${ }^{[4]}$.

\subsection{Buried pipe system is a widely used and feasible choice}

It can store heat in the stable shallow surface, and it is also an excellent heat and cold source of soil coupled heat pump. The study of this system should pay attention to the influence of different layout structure and soil environment on heat storage efficiency.

Basing on the principle of linear heat source and 
energy conservation, Yan Lijun et al. derived the expression of heat action radius in a certain error range, and studied the temperature variation characteristics of soil around u-tube with constant inlet temperature and variable heat flow boundary by numerical simulation ${ }^{[5]}$.

The thermal stratification and thermal insulation material of the thermal storage tank are the research hotspots. The experimental results show that the seasonal thermal storage efficiency of the system reached about $60 \%$ from 1997 to $2003^{[6]}$.

\section{Technology optimization of low temperature heat storage under industrial drying demand}

\subsection{An improved case based on drying de- mand}

In the field of food processing and wood supply industry, drying process is very important and can not be neglected. However, the traditional electric heating drying and air supply drying methods often need a lot of energy. Especially in the field of wood processing, drying is becoming the most energy-consuming process. The solar drying and heat pump drying methods have solved this problem. Researchers at home and abroad have carried out a large number of studies, of which the phase change heat storage research is more in-depth and mature.

The application of phase change material (PCM) has greatly improved the energy-saving efficiency of the system. Wang Jianfeng et al. compared the efficiency of heat storage with drying system and the efficient of systems without drying system, and found that phase change material (PCM) can save at least 20\% energy than the original system in the later stage of drying ${ }^{[7]}$. Gan Xuefei et al. have carried out systematic experiments to compare the effectiveness of paraffin heat storage systems in different weather conditions ${ }^{[8]}$.

In the field of solar drying, most of the heat storage systems researches are based on phase change heat storage technique. Considering the need for temperature and cost saving, the most widely used heat storage medium is paraffin, and many heat storage devices which are based on this medium have been developed at home and abroad.

Feng Xiaojiang et al. developed a set of heat storage device for wood drying, using solar energy for energy supplying, storing heat in a heat storage system when the sunshine is plenty ${ }^{[9]}$. Huang Jijie et al. developed a dryer, The heat storage structure is a box structure with heat conducting copper pipes in it, and the paraffin is filled in the gap as a heat storage material ${ }^{[10]}$.

The heat storage tank, a relatively basic heat storage device, has also been quite innovated in the industrial drying field. Zhang Li et al. (solar energy water source heat pump drying system) has developed a combined drying system of solar energy and heat pump. The heat storage tank, which is essentially a heat exchanger, is selected for heat storage. However, the heat storage tank is innovatively upgraded into a low-temperature water tank and a high-temperature water tank. In addition, a part of the hot water in the heat storage tank is used for heating the refrigerants of the air source heat pump, which makes the system far more effective than heating with air $^{[11]}$.

\subsection{Summary of improvement ideas}

The essence of industrial drying determines that it has higher requirements for heat storage craft. Recent innovations in this field for heat storage systems include but not limited to the above contents. As can be seen from the above examples, thermal storage systems are improved to match the function of the whole drying system, which is the criterion of the thermal storage system and even most of the science and technology.

\section{Conclusion}

After carding the research results and technical innovation of heat storage technology under various industrial demands, we can draw the conclusion that the heat storage technology is becoming increasingly mature. It can be seen that different emphasis on heat storage methods combined with the use of the environment there will be a variety of technological innovation when different emphasis on heat storage methods are combined with the use of the environment.

However, the main problems in the field of low-temperature heat storage now is still how to further improve the heat storage efficiency, and how to improve the efficiency while reducing the cost as much as possible. The above examples of innovation coalesced with the actual industrial requirements also provide inspira- 
tion for further research. And it is the knowledge of tank design for the inclined temperature layer that makes the heat storage technology in this field mature.

\section{References}

1. Liang L, Chen M, Duan Li, et al. Research progress of heat storage technology in the field of solar thermal power generation and cogeneration (in Chinese). Thermal Power Generation 2020; 49(3): 8-15.

2. Han J. Study on thermal storage characteristics of single tank in oblique thermal layer for solar thermal power generation (in Chinese). North China Electric Power University; 2015.

3. Zhang Y. New materials for heat storage and transfer - Brief introduction of functional thermal fluids (in Chinese). Chinese Journal of Nature 1998; (1): 3-5.

4. Zhao X, Zhao Y, Wang J, et al. Research progress of solar energy interseasonal heat storage technology (in Chinese). Advances in New and Renewable Energy 2017; 5(1): 73-80.

5. Yan Li, Zhang X. Study on heat storage characteristics of buried pipe heat exchanger based on heat ac- tion radius (in Chinese). Refrigeration Technique 2015; 35(1): 1-5+10.

6. Wang E, Qi C, Yang H, et al. Experimental analysis of solar energy heating system and soil heat storage in buildings with interseasonal heat storage (in Chinese). In: Proceedings of the 2007 Annual Conference of China Refrigeration Society; 2007; Chinese Association of Refrigeration; 2007. p. 357-361.

7. Wang J, Ouyang Y, Zhu Y, et al. Experimental study on the application of phase change materials in heat pump drying (in Chinese). Journal of Solar Energy 2002; (1): 22-26.

8. Gan X. Study on the application of paraffin heat storage system in solar wood drying (in Chinese). Beijing Forestry University; 2012.

9. Feng X. Design and performance research of phase change storage solar energy wood drying unit (in Chinese). Beijing Forestry University; 2012.

10. Huang J. Design and flow field analysis of solar drier containing heat storage system (in Chinese). Hefei University of Technology; 2019.

11. Zhang L. Solar water source heat pump combined drying system and process optimization (in Chinese) Beijing Forestry University; 2015. 